\title{
AMBIDEXTROUS CHIRAL DOMAINS IN NONCHIRAL LIQUID-CRYSTALLINE MATERIALS*
}

\author{
Lech LongA ${ }^{\dagger}$, Karol Trojanowski
}

The Marian Smoluchowski Institute of Physics

Division of Statistical Physics

and

The Mark Kac Complex Systems Research Center

Jagiellonian University, Reymonta 4, 30-059 Kraków, Poland

(Received May 20, 2013)

Recently, we studied equilibrium properties of the Lebwohl-Lasher model with quadrupolar and octupolar interactions in the large twist limit. A complete mean field analysis of the model and Monte Carlo simulations were presented to show a global stabilization of new structures like tetrahedratic, nematic tetrahedratic, and chiral nematic tetrahedratic phases of $T_{d}, D_{2 d}$, and $D_{2}$ symmetry, respectively. Here, by means of Monte Carlo simulation on two-dimensional system, we show that the model can also give a molecular interpretation of macroscopic regions with opposite optical activity (ambidextrous chirality), observed in achiral bent-core systems, and recently in ferrocenomesogens and flexible liquid crystal dimers. The resulting superstructures include short- and long-range twist deformations.

DOI:10.5506/APhysPolB.44.1201

PACS numbers: $61.30 . \mathrm{Cz}, 64.70 . \mathrm{mf}, 05.70 .-\mathrm{a}$

Complex supramolecular structures formed by bent-core (banana-shaped) systems [1-4], superstructures found in ferrocenomesogens [5] and in nematic liquid crystals formed by flexible dimers [6-8] can exhibit unusual properties, unknown to conventional mesogenic materials. The most striking is the occurrence of a macroscopic spontaneous chiral order forming domains of opposite optical activity. This observation is unusual in the sense that chirality and chiral structures of liquid crystals have so far been regarded as resulting from the presence of optically active molecules (e.g. [9-11]), while

* Presented at the XXV Marian Smoluchowski Symposium on Statistical Physics, "Fluctuation Relations in Nonequilibrium Regime", Kraków, Poland, September 10-13, 2012.

$\dagger$ lech.longa@uj.edu.pl 
all the molecules that we mentioned above are intrinsically achiral. Interestingly, spontaneous formation of macroscopic chiral domains of opposite handedness (ambidextrous chirality) was observed not only in more ordered smectic phases but also in ordinary isotropic and nematic phases.

Lubensky and Radzihovsky [12] argued that chiral order of non-chiral materials can be explained at fundamental, symmetry level by considering at least quadrupolar (biaxial) and octupolar (tetrahedratic) tensor order parameters. They presented exhaustive list of possible liquid crystalline phases along with their symmetries that result from this hypothesis and cataloged the universality classes of the corresponding phase transitions.

The molecular level studies, although still scarce, support relevance of the molecular quadrupolar interactions in the microscopic description of the systems considered [13-23]. It is also relatively easy to show that rigid molecules of $C_{2}$-symmetry, which approximate the shape of bent-core molecules, generate quadrupolar and octupolar contributions to the Onsager's excluded volume [24].

In our recent papers [25, 26], we introduced a generalized Lebwohl-Lasher model with competing dispersion quadrupolar (uniaxial and biaxial) and octupolar (tetrahedratic) interactions to study a possibility of spontaneous chiral symmetry breaking. We assumed that liquid-crystalline molecules (or molecular complexes) interact with the $\mathcal{O}(3)$-invariant pair potential of the form

$$
V(i, j)=-\epsilon\left(r_{i j}\right)\left[\boldsymbol{Q}\left(\boldsymbol{\Omega}_{i}\right) \cdot \boldsymbol{Q}\left(\boldsymbol{\Omega}_{j}\right)+\tau \boldsymbol{T}_{2}^{3}\left(\boldsymbol{\Omega}_{i}\right) \cdot \boldsymbol{T}_{2}^{3}\left(\boldsymbol{\Omega}_{j}\right)\right],
$$

where $r_{i j}=\left|\boldsymbol{r}_{i j}\right|$ is the distance between molecular centers of mass and where $\boldsymbol{Q}=\boldsymbol{T}_{0}^{2}+\sqrt{2} \lambda \boldsymbol{T}_{2}^{2}$ is the second-rank quadrupolar tensor, defined with respect to two-fold axes of the molecular quadrupole moment. The $\boldsymbol{T}_{2}^{3}$-term takes into account interactions between molecular octupolar, third-rank tensors. The three tensors $\boldsymbol{T}_{m}^{L}$ of $L=2,3$ are given by

$$
\begin{aligned}
& \boldsymbol{T}_{0}^{2}\left(\boldsymbol{\Omega}_{k}\right)=\sqrt{\frac{3}{2}}\left(\boldsymbol{c}_{k} \otimes \boldsymbol{c}_{k}-\frac{1}{3} \mathbf{1}\right), \\
& \boldsymbol{T}_{2}^{2}\left(\boldsymbol{\Omega}_{k}\right)=\frac{1}{\sqrt{2}}\left(\boldsymbol{a}_{k} \otimes \boldsymbol{a}_{k}-\boldsymbol{b}_{k} \otimes \boldsymbol{b}_{k}\right), \\
& \boldsymbol{T}_{2}^{3}\left(\boldsymbol{\Omega}_{k}\right)=\frac{1}{\sqrt{6}} \sum_{(\boldsymbol{x}, \boldsymbol{y}, \boldsymbol{z}) \in \pi\left(\boldsymbol{a}_{k}, \boldsymbol{b}_{k}, \boldsymbol{c}_{k}\right)} \boldsymbol{x} \otimes \boldsymbol{y} \otimes \boldsymbol{z},
\end{aligned}
$$

where $\boldsymbol{\Omega}_{k}$ is the orthonormal tripod of vectors $\left\{\boldsymbol{a}_{k}, \boldsymbol{b}_{k}, \boldsymbol{c}_{k}\right\}$ attached to the $k$ th molecule or molecular complex. Summation in $\boldsymbol{T}_{2}^{3}$ runs over six permutations of $\boldsymbol{a}_{k}, \boldsymbol{b}_{k}, \boldsymbol{c}_{k}$. Finally, the scalar product '. ' is defined as a full contraction over Cartesian indices. 
For $\tau=\lambda=0$, the model (1) accounts for the first-order isotropic to uniaxial nematic phase transition [28]. The case of $\lambda \neq\{0, \sqrt{3 / 2}\}$ serves as a generic model of a biaxial nematic order [17, 29]. The model with purely tetrahedratic coupling, proportional to $\epsilon \tau$, shows the formation of absolutely stable tetrahedratic phase of global $T_{d}$ symmetry. It was studied by Fel [30, 31], Romano [32], and by us [25, 26].

In the pair-interaction potential (1), the $k$ th molecule degrees of freedom, $\Omega_{k}$, involve a proper rotation $\boldsymbol{\Omega}^{\prime}{ }_{k}$, expressed e.g. in terms of standard Euler angles $\left(\alpha_{k}, \beta_{k}, \gamma_{k}\right)$ and an inversion operation of the molecule-fixed system of frame, referred to as a parity degree of freedom $p_{k}=\boldsymbol{a}_{k} \cdot\left(\boldsymbol{b}_{k} \times \boldsymbol{c}_{k}\right)= \pm 1$. That is

$$
\boldsymbol{\Omega}_{k}=\left\{p_{k}, \boldsymbol{\Omega}^{\prime}{ }_{k}\right\}, \quad \underset{(k)}{\operatorname{Tr}}=\frac{1}{2} \sum_{p_{k}= \pm 1} \int d \boldsymbol{\Omega}^{\prime}{ }_{k} .
$$

Clearly, the inversions change sign of $\boldsymbol{T}_{2}^{3}$, but leave $\boldsymbol{Q}$ unaffected.

The combination of biaxial and tetrahedratic interactions, Eq. (1), studied in [25-27], leads to a formation of new structures like a tetrahedratic nematic phase of $D_{2 d}$ symmetry, and, most importantly, a chiral tetrahedratic nematic of $D_{2}$ symmetry. The chiral phase is stabilized as a result of coupling between octupolar and quadrupolar biaxial long-range orders and is absent in the uniaxial limit $\left(\lambda=0\right.$ or $\left.\lambda=\sqrt{\frac{3}{2}}\right)$. In the nematic tetrahedratic phase, weakly biaxial left- and right-handed homochiral domains are formed, but their chirality mutually cancels out and the overall structure is nonchiral. The studies were carried out with neglect of chirality-induced spatial modulation of the orientational structures, which was regarded as secondary characteristic.

The class of spatially homogeneous, orientational structures identified in $[25,26]$ becomes unstable against spontaneous twist formation (short- or long-ranged) if we supplement Eq. (1) with further couplings that involve $\boldsymbol{T}$ tensors, Eq. (2), and the intermolecular unit vector $\hat{\boldsymbol{r}}_{i j}=\boldsymbol{r}_{i j} / r_{i j}$. The simplest interaction, linear in $\hat{\boldsymbol{r}}_{i j}$, that can be added to Eq. (1), reads [26]

$$
\begin{aligned}
V_{c}\left(p_{i},{\boldsymbol{\boldsymbol { } ^ { \prime }}}_{i}, p_{j}, \boldsymbol{\Omega}^{\prime}{ }_{j}\right)= & \frac{\kappa}{\epsilon}\left[\Theta_{\alpha \beta \gamma}\left(\boldsymbol{\Omega}_{i}\right) Q_{\alpha \nu}\left(\boldsymbol{\Omega}_{i}\right) Q_{\beta \nu}\left(\boldsymbol{\Omega}_{j}\right)\right. \\
& \left.-\Theta_{\alpha \beta \gamma}\left(\boldsymbol{\Omega}_{j}\right) Q_{\alpha \nu}\left(\boldsymbol{\Omega}_{j}\right) Q_{\beta \nu}\left(\boldsymbol{\Omega}_{i}\right)\right]\left(\hat{\boldsymbol{r}}_{i j}\right)_{\gamma},
\end{aligned}
$$

where $\left(\hat{\boldsymbol{r}}_{i j}\right)_{\gamma}=-\left(\hat{\boldsymbol{r}}_{j i}\right)_{\gamma}$ is the $\gamma$ component of the intermolecular unit vector and where we have used the Einstein convention for the repeated indices. The third-rank molecular tensor $\Theta_{\alpha \beta \gamma}$, totally antisymmetric in $\{\alpha, \beta, \gamma\}$, is given by 


$$
\Theta_{\alpha \beta \gamma}=2 \sqrt{2} \sum_{(x, y, z) \in c\{\alpha, \beta, \gamma\}} T_{0, x \mu}^{2} T_{2, y \nu}^{2} T_{2, \mu \nu z}^{3},
$$

where summation runs over cyclic permutations of $\{\alpha, \beta, \gamma\}(c\{\alpha, \beta, \gamma\}=$ $\{(\alpha, \beta, \gamma),(\gamma, \alpha, \beta),(\beta, \gamma, \alpha)\})$.

The modified pair interaction potential, $V_{\text {total }}$, is sum of $V$ and $V_{c}$, Eqs. (1), (4)

$$
V_{\text {total }}\left(p_{i}, \boldsymbol{\Omega}^{\prime}{ }_{i}, p_{j}, \boldsymbol{\Omega}^{\prime}{ }_{j}, \hat{\boldsymbol{r}}_{i j}\right)=V\left(p_{i}, \boldsymbol{\Omega}^{\prime}{ }_{i}, p_{j}, \boldsymbol{\Omega}^{\prime}{ }_{j}\right)+V_{c}\left(p_{i}, \boldsymbol{\Omega}_{i}^{\prime}, p_{j}, \boldsymbol{\Omega}^{\prime}{ }_{j}, \hat{\boldsymbol{r}}_{i j}\right) .
$$

Note that

$$
V_{\text {total }}\left(p_{i}, \boldsymbol{\Omega}^{\prime}{ }_{i}, p_{j}, \boldsymbol{\Omega}^{\prime}{ }_{j}, \hat{\boldsymbol{r}}_{i j}\right)=V_{\text {total }}\left(-p_{j}, \boldsymbol{\Omega}^{\prime}{ }_{j},-p_{i}, \boldsymbol{\Omega}^{\prime}{ }_{i}, \hat{\boldsymbol{r}}_{i j}\right),
$$

which, along with $V_{c} \sim\left(p_{i}+p_{j}\right)$, allows to take $\kappa \geq 0$. Additionally, the $V_{c}$-term is relevant, given that $p_{i}=p_{j}$, and vanishes for states of opposite parity $\left(p_{i}=-p_{j}\right)$.

The properties of $V_{c}$ imply that for nonvanishing $\kappa$ the chiral nematic tetrahedratic structure becomes unstable against long-range spontaneous twist formation, while short-ranged twisted configurations of opposite chirality $\left(\left\langle p_{i}\right\rangle=0\right)$ are formed in non-chiral phases. To prove this statement, we perform Monte Carlo simulations for the model (6). For simplicity, we assume the molecules to occupy the sites of a two-dimensional square lattice, but later will argue that qualitative results should also hold for threedimensional systems. The strength of the interaction is given by $\epsilon\left(r_{i j}\right)$, taken to be a positive constant $\epsilon$ when particles $i$ and $j$ are nearest neighbors and zero otherwise. We carry out the simulations on a square lattice of relatively large size $64 \times 64$ with periodic boundary conditions, using Metropolis algorithm. The orientational degrees of freedom of molecules are coded in a quaternion representation. Each attempted MC move involves the proper rotation of a molecule's orientation and parity inversion. The size of the MC rotational step is adjusted to give an acceptance ratio between $30 \%$ and $40 \%$ in the ordered phases.

The results from typical simulations are shown in Figs. 1-3. For relatively low values of $\kappa=1$, the low-temperature chiral tetrahedratic nematic phase is replaced by the cholesteric-like (homochiral) phase. At high temperatures, the cholesteric order breaks down and the short-range domains of opposite chirality are formed, as observed experimentally in nematic and isotropic phases. The result should be independent of the space dimension except that a more complex structures, like Blue Phases, may form for three dimensional lattices. To see this, let us consider the ground state properties of (6) for $\epsilon>0$ and $\tau>0$. For $\kappa=0$, we find that $\operatorname{Min}_{\left\{\Omega^{\prime}\right\}} V_{\text {total }}(i, j)=-\epsilon\left(1+2 \lambda^{2}+p_{i} p_{j} \tau\right)$, 
which is satisfied by configurations of $p_{i}=p_{j}$, where twofold axes of $\boldsymbol{Q}(i)$ $\left(\boldsymbol{T}_{2}^{3}(i)\right)$ and $\boldsymbol{Q}(j)\left(\boldsymbol{T}_{2}^{3}(j)\right)$ are aligned parallel to each other. This local arrangement can be extended globally over the whole lattice (irrespective of the space dimension) to stabilize (at $T=0$ for one-dim systems) the chiral nematic tetrahedratic structure.

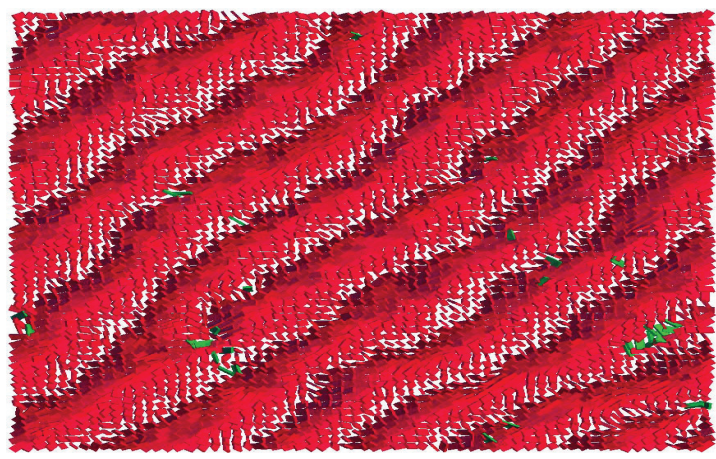

Fig. 1. (Color online) Snapshot of the molecular equilibrium organization obtained from $\mathrm{MC}$ simulations for the two-dimensional square lattice $(64 \times 64)$, generated by the interaction potential (6). Parameters taken are $\lambda=0.3, \tau=\kappa=1$ and reduced temperature $t=k_{\mathrm{B}} T / \epsilon=0.55$. Bricks are oriented along eigenvectors of the molecular $\boldsymbol{Q}$-tensors; arms are proportional to absolute values of the corresponding eigenvalues. The associated octupolar order is not shown. Note that highly ordered cholesteric-like phase with a single dislocation is formed. It replaces homochiral tetrahedratic nematic, stable for $\kappa=0$.

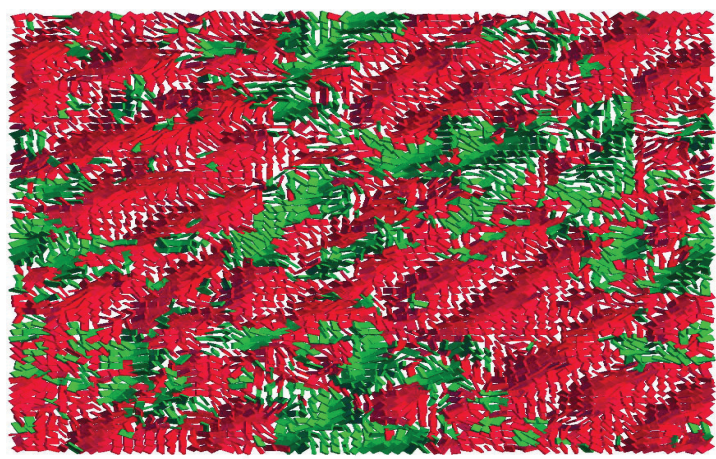

Fig. 2. (Color online) Snapshot of the molecular equilibrium configuration obtained from MC simulations defined in captions to Fig. 1 for $t=k_{\mathrm{B}} T / \epsilon=0.65$. The structure is still cholesteric-like, but with many, short-range domains of opposite chirality (coded with green/light gray color). 


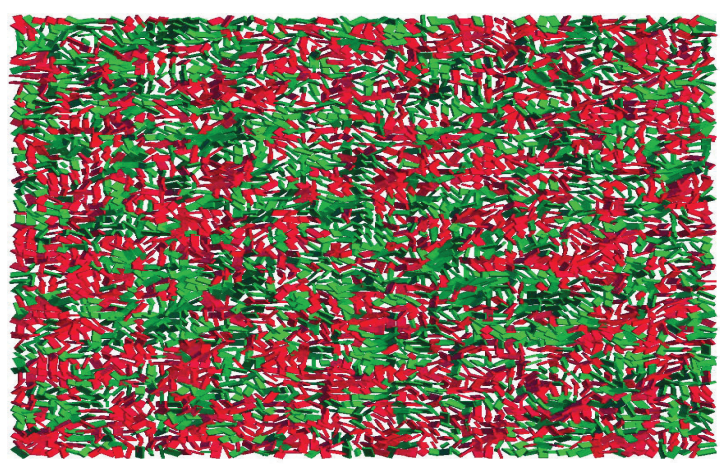

Fig. 3. (Color online) Snapshot of the molecular equilibrium organization obtained from $\mathrm{MC}$ simulations defined in captions to Fig. 1 for $t=k_{\mathrm{B}} T / \epsilon=1$. The structure is isotropic with many short-range domains of opposite chirality (coded with green/light gray and red/gray colors). Note that phase is overall non-chiral.

For $\kappa \neq 0$ and $\lambda$ giving the biaxial, molecular tensor $\boldsymbol{Q}$, the ground state configuration corresponds to $\boldsymbol{a}_{i}, \boldsymbol{a}_{j}$ and $\hat{\boldsymbol{r}}_{i j}$ being parallel to each other. The perpendicular axes $\left\{\boldsymbol{b}_{j}, \boldsymbol{c}_{j}\right\}$ are rotated by $\beta_{i j}$ about $\boldsymbol{a}_{i}$ clockwise $\left(p_{i}+p_{j}=2\right)$ or counterclockwise $\left(p_{i}+p_{j}=-2\right)$ from $\left\{\boldsymbol{b}_{i}, \boldsymbol{c}_{i}\right\}$, where

$$
\tan \left(2 \beta_{i j}\right)=\frac{\kappa \Lambda\left(p_{i}+p_{j}\right)}{\Lambda+4 \tau}=\frac{2 \kappa \Lambda p_{i}}{\Lambda+4 \tau},
$$

and where

$$
\Lambda=3+2 \lambda(\sqrt{6}+\lambda) .
$$

The equation (8) predicts molecular twist of two neighboring molecules in the ground state and allows to estimate the average molecular twist $\langle\beta\rangle$ of the nearest-neighbor tripods for not too high temperatures as [26]

$$
\langle\beta\rangle \approx=\frac{\kappa \Lambda}{\Lambda+4 \tau}\langle p\rangle .
$$

Both of the above equations support our previous statement that superstructures with twist should be observed independent of space dimensionality. However, the simulations for three dimensional lattice may give additional stable structures with twist propagating in more than one direction. Analysis of this more complex case is postponed to our forthcoming publication.

The work has been supported by the International Ph.D. Projects Programme of the Foundation for Polish Science within the European Regional Development Fund of the European Union, agreement No. MPD/2009/6. 


\section{REFERENCES}

[1] R.A. Reddy, C. Tschierske, J. Mater. Chem. 16, 907 (2006).

[2] H. Takezoe, Y. Takanishi, Jpn. J. Appl. Phys. 45, 597 (2006).

[3] O. Francescangeli et al., Soft Matter 7, 895 (2011).

[4] S.D. Peroukidis, A.G. Vanakaras, D.J. Photinos, Phys. Rev. E84, 010702(R) (2011).

[5] E.H. Kim, O.N. Kadkin, S.Y. Kim, M.G. Choi, Eur. J. Inorg. Chem. 2011, 2933 (2011).

[6] V. Görtz et al., Soft Matter 5, 463 (2009).

[7] V.P. Panov et al., Phys. Rev. Lett. 105, 167801 (2010).

[8] M. Cestari et al., Phys. Rev. E84, 031704 (2011).

[9] See e.g., P.G. de Gennes, J. Prost, The Physics of Liquid Crystals, Second Edition, Clarendon Press, Oxford 1993.

[10] J. Englert, L. Longa, H. Stark, H.-R. Trebin, Phys. Rev. Lett. 81, 1457 (1998).

[11] L. Longa, M. Cieśla, H.-R. Trebin, Phys. Rev. E67, 061705 (2003).

[12] T.C. Lubensky, L. Radzihovsky, Phys. Rev. E66, 031704 (2002).

[13] J. Peláez, M. R.Wilson, Phys. Rev. Lett. 97, 267801 (2006).

[14] M.S. Park et al., Phys. Rev. Lett. 105, 027801 (2010).

[15] P. Grzybowski, L. Longa, Phys. Rev. Lett. 107, 027802 (2011).

[16] M.A. Osipov, G. Pająk, J. Phys.: Condens. Matter 24, 142201 (2012).

[17] L. Longa, P. Grzybowski, S. Romano, E. Virga, Phys. Rev. E71, 051714 (2005).

[18] L. Longa, G. Pająk, T. Wydro, Phys. Rev. E76, 011703 (2007).

[19] D. Allender, L. Longa, Phys. Rev. E78, 011704 (2008).

[20] M. Ciesla, L. Longa, Mol. Cryst. Liq. Cryst. 545, 214 (2011).

[21] Y. Jang et al., Appl. Phys. Lett. 95, 183304 (2009).

[22] H.G. Yoon et al., Phys. Rev. E81, 051706 (2010).

[23] A.D. Buckingham, Discuss. Faraday Soc. 40, 232 (1965).

[24] F. Bisi, L. Longa, G. Pająk, R. Rosso, Mol. Cryst. Liq. Cryst. 525, 12 (2010).

[25] L. Longa, G. Pająk, T. Wydro, Phys. Rev. E79, 040701 (2009).

[26] K. Trojanowski, G. Pająk, L. Longa, T. Wydro, Phys. Rev. E86, 011704 (2012).

[27] L. Longa, G. Pająk, Mol. Cryst. Liq. Cryst. 541, 152 (2011).

[28] P.A. Lebwohl, G. Lasher, Phys. Rev. A6, 426 (1972).

[29] F. Biscarini et al., Phys. Rev. Lett. 75, 1803 (1995).

[30] L.G. Fel, Phys. Rev. E52, 702 (1995).

[31] L.G. Fel, Phys. Rev. E52, 2692 (1995).

[32] S. Romano, Phys. Rev. E77, 021704 (2008). 